\title{
Accuracy of automatic deformable structure propagation for high-field MRI guided prostate radiotherapy
}

\author{
Rasmus Lübeck Christiansen ${ }^{1,2^{*}}$ D, Lars Dysager ${ }^{3}$, Anders Smedegaard Bertelsen², Olfred Hansen ${ }^{1,3}$, \\ Carsten Brink ${ }^{1,2}$ and Uffe Bernchou ${ }^{1,2}$
}

\begin{abstract}
Background: In this study we have evaluated the accuracy of automatic, deformable structure propagation from planning CT and MR scans for daily online plan adaptation for MR linac (MRL) treatment, which is an important element to minimize re-planning time and reduce the risk of misrepresenting the target due to this time pressure.

Methods: For 12 high-risk prostate cancer patients treated to the prostate and pelvic lymph nodes, target structures and organs at risk were delineated on both planning MR and $C T$ scans and propagated using deformable registration to three T2 weighted MR scans acquired during the treatment course. Generated structures were evaluated against manual delineations on the repeated scans using intra-observer variation obtained on the planning MR as ground truth.
\end{abstract}

Results: MR-to-MR propagated structures had significant less median surface distance and larger Dice similarity index compared to CT-MR propagation. The MR-MR propagation uncertainty was similar in magnitude to the intra-observer variation. Visual inspection of the deformed structures revealed that small anatomical differences between organs in source and destination image sets were generally well accounted for while large differences were not.

Conclusion: Both CT and MR based propagations require manual editing, but the current results show that MR-to-MR propagated structures require fewer corrections for high risk prostate cancer patients treated at a high-field MRL.

Keywords: MR-linac, Radiotherapy, Prostate cancer, Computed tomography, Magnetic resonance, Deformable image registration, Treatment precision

\section{Background}

Changes in anatomy over a radiotherapy (RT) treatment course for pelvic cancer has motivated adaptive treatment schemes $[1,2]$. However, until now the limitations of cone beam CT (CBCT) image quality and soft tissue contrast has hampered the clinical implementation [3, $4]$. With the introduction of magnetic resonance (MR) radiotherapy delivery systems, daily, MR guided adaptive radiotherapy (ART) has become possible with the potential to reduce the safety margins used today [5-7] and

\footnotetext{
* Correspondence: rasmus.lubeck.christiansen@rsyd.dk

1 Department of Clinical Research, University of Southern Denmark, Winsløwparken 19 3. Sal, 5000 Odense C, Denmark

2Laboratory of Radiation Physics, Department of Oncology, Odense University Hospital, Kløvervænget 19, Indgang 85, Pavillion, Stuen, 5000 Odense C, Denmark

Full list of author information is available at the end of the article
}

thus the treatment toxicity [8]. However, the workflow for an MR guided treatment fraction lasts longer than standard CBCT based linac treatment workflow, which increases the risk of patient motion during the treatment session [9-11]. One of the most time consuming steps in the MR workflow is the re-delineation and validation of target structures and organs at risk (OAR) [10, 12]. Automatic propagation of structures might also reduce the risk of misdelineations that could introduce systematic or large random errors. Two commercial MR linac (MRL) systems are currently clinically available and for both systems a deformable image registration (DIR) algorithm is part of the treatment planning system (TPS) to facilitate a fast deformation of the planning images and structure propagation to reduce this time.

(c) The Author(s). 2020 Open Access This article is distributed under the terms of the Creative Commons Attribution 4.0 International License (http://creativecommons.org/licenses/by/4.0/), which permits unrestricted use, distribution, and reproduction in any medium, provided you give appropriate credit to the original author(s) and the source, provide a link to the Creative Commons license, and indicate if changes were made. The Creative Commons Public Domain Dedication waiver (http://creativecommons.org/publicdomain/zero/1.0/) applies to the data made available in this article, unless otherwise stated. 
The standard workflow suggested by the vendor for the high field MRL has been specified as propagation of structures from the planning CT scan (pCT) to the MR scan of the given treatment session. Previously, different commercial algorithms have been evaluated on different anatomies when performing DIR from CT to MR, showing great potential, but also uncertainties in the deformations [13-16]. Hence, manual revision and some manual corrections of the propagated structures would usually be required across the different commercial DIR solutions and anatomies [13, 17-19]. Therefore use of MR-MR DIR in the online MRL treatment workflow, as described by Bertelsen et al. [11], could provide more precisely propagated structures. However, to our knowledge, this has not yet been verified.

It has been demonstrated that manual delineation of soft tissue structures is more consistent when using MR rather than $\mathrm{CT}$, both in terms of the inter- and intraobserver variation [20-22]. E.g. Smith et al. showed that the inter-observer variation of prostate on T2 weighted (T2w) MRI was smaller than on CT [20]. Furthermore, the volume of the prostate delineated on CT was larger than on MR [20, 22]. For these reasons the intraobserver variation on MR has been regarded as the reference precision in clinical practice $[20,21,23]$.

This study investigates the geometric accuracy of deformable image registration of target volumes and OAR in high-risk prostate cancer patients for both CT-MR and MR-MR registrations using manual delineations as ground truth.

\section{Methods}

\section{Patients}

Twelve high-risk prostate cancer patients referred for 78 Gy for the prostate and proximal part of the seminal vesicles (SV) and 56 Gy for the pelvic lymph nodes, both delivered in 39 fractions on standard CBCT linacs, were included in the study. Exclusion criteria were contraindications to MR and metal implants in the pelvis (e. g. prosthetic hips).

The study has been approved by the regional board of ethics and all included patients have given their signed consent to participate.

\section{Image acquisition}

Planning CT scans were acquired on either a Philips Big Bore Brilliance 16 slice scanner (Philips Medical Systems BV, The Netherlands) or a Toshiba Aquillion One (Canon Medical Systems Corporation, Japan). The scan parameters applied were: $50 \mathrm{~cm}$ field of view (FOV), $512 \times 512$ matrix and $3 \mathrm{~mm}$ slice thickness.

All MR scans were performed in treatment position on a Philips Ingenia 1.5 T (Philips Medical Systems BV, The Netherlands) equipped with a flat table top and fixation device for feet and knees. Supplementary to a planning MR (pMR) acquired immediately before or after the pCT, three sets of MRI $\left(\mathrm{MR}_{10}, \mathrm{MR}_{20}\right.$ and $\mathrm{MR}_{30}$ ) were acquired at the 10th, 20th, and 30th fraction (allowing a variation of $+/-2$ days) as representative samples of daily MRI sessions over their treatment course. The T2w sequence applied in this study was a 3D scan with 3D image distortion correction applied. The following parameters were used to resemble the sequence suggested for use at the MRL by the vendor: 300 slices, $400 \times 400 \mathrm{~mm}$ FOV, $0.5 \times 0.5 \mathrm{~mm}$ pixels, 1 mm slice thickness, TE $216 \mathrm{~ms}$, TR $1800 \mathrm{~ms}$. Scan time was $5.56 \mathrm{~min}$.

The geometric fidelity of the MR scanner was evaluated weekly according to a quality assurance program including scans and analysis of a vendor specific phantom. MR sequences applied clinically were evaluated with a MagPhan RT 820 phantom (The Phantom Laboratory, Salem, NY, USA) scan analysed in Total QA software (Image Owl, Greewich, NY, USA).

\section{Structure delineation}

For the current study, target volumes (prostate, SV and CTV56) were delineated on pCT and each acquired T2w $\mathrm{MR}$ set ( $\mathrm{pMR}, \mathrm{MR}_{10}, \mathrm{MR}_{20}$ and $\mathrm{MR}_{30}$ ) by one experienced oncologist. The prostate structure included the connective tissue capsule surrounding the gland. The elective volume CTV56 was defined as pelvic lymph nodes with a $7 \mathrm{~mm}$ margin as described by RTOG consensus guidelines [24]. All OAR (rectum, bladder, penile bulb, bowel and bilateral femoral heads) were delineated on each image set by one experienced RTT trained in delineation. For delineations on pCT a standard abdominal window/level settings were used (w 350/ 1 40). Individual window/level settings were used for MRI delineations. Previous image sets were available for the observer during contouring, as they would be in a clinical setting.

These manual delineations represent the ground truth as to what each structure should encompass. The current study focuses on differences due to use of different image modality and not inter-observer variations. However the observed uncertainties are compared to the intra-observer variation which is less than the interobserver variation. Thus, structures were re-delineated on all patients' pMR by the same observers no less than one month after initial delineation to determine the intra-observer variation. In lack of the absolute truth, the intra observer-variation represents the best accuracy we can expect from the propagated structures [18].

Delineations were performed in the treatment planning system (TPS) dedicated to the Elekta Unity high-field MRL, Monaco ver. 5.40 (Elekta AB, Stockholm, 


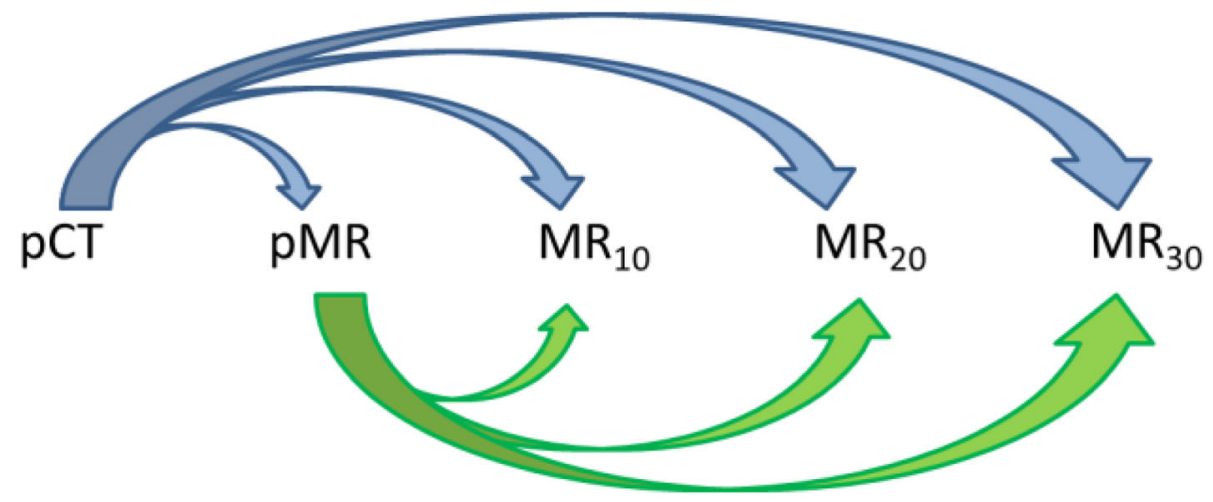

Fig. 1 Schematic representation of image deformation and subsequent structure propagation from planning scans performed in the TPS
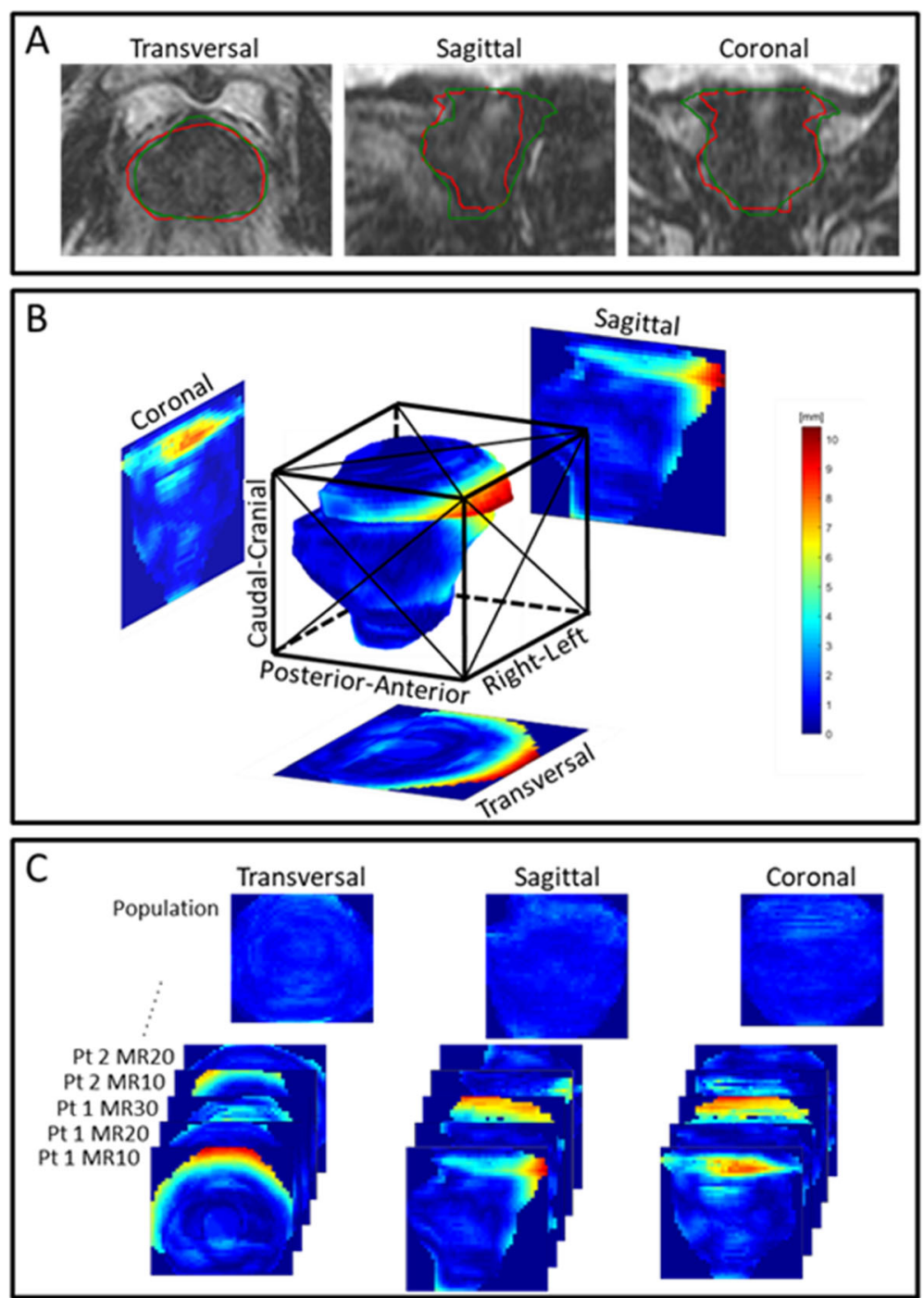

Fig. 2 The manually delineated prostate (green) is defined as reference to the deformed prostate (red) are shown in a. Distance between the two is calculated in $\mathbf{b}$, and projections onto the coronal, sagittal and transversal planes are made. In $\mathbf{c}$, the projections are per projection summed over the patients to provide the population percentile surface distance projection image 
Sweden). In this TPS, images are displayed using pixel interpolation.

\section{Image registration}

All registrations and structure propagations were performed in Monaco. The $\mathrm{pCT}$ was registered to each MR set and the pMR to each additional MR, as illustrated schematically in Fig. 1.

Standard settings of the commercial deformation software were used in all cases, specific details of the algorithm is not disclosed by the vendor. Deformation of CT images to MR was performed using normalized mutual information, whereas MR-MR deformations were performed using a local cross correlation algorithm.

\section{Structure comparison}

Three metrics were calculated to evaluate the accuracy of DIR: The Dice similarity coefficient (DSC), which delivers the ratio of overlap between the manually delineated structure and the corresponding deformable propagated structure [25]. This measure is most relevant for smaller structures, as the index for large structures might be very high, although large clinically relevant volumes might not overlap. The mean surface distance (MSD), as described by Zukauskaite et al. [26] gives the average distance between the manual and deformed structure in absolute measures, which is particularly relevant for larger structures. The Hausdorff distance (HD) delivers the greatest distance between a given pair of structures to show a worst case scenario [27], thus very sensitive to outliers in the data. For each patient the average value over all the scans of the DSC, MSD and HD was calculated for each structure investigated for both MR-MR and CT-MR registrations and compared to the intra-observer variation. In order to assess spatial patterns in the distribution of variation between ground truth and deformed or re-delineated contours, population based surface distance projection images were generated in the transversal, sagittal, and coronal plane for each structure. These projection images, showing the differences between ground truth and deformed structures or intra-observer variation, were created using the following procedure (see Fig. 2 for a graphical overview): The smallest possible bounding box, oriented along the main patient directions (Anterior-Posterior, Right-Left, and Cranio-Caudal), surrounding a given patient organ was initially defined. For all voxels at the surface of the organ, the distance to the reference organ was measured and then projected into the sagittal, transversal, and coronal plane. In these three planes, a grid encompassing the bounding box and with fixed number of pixels was defined (Additional file 1: Table A1). Within each pixel the projected deviations was averaged in order to observe scan specific spatial structures within the uncertainty. To provide the population based surface distance projection images, the 50 as well as the 90 percentile of all the scan individual projection deviations were subsequently found in each pixel. Although a specific type of structure may have varying size and shape depending on scan and patient, fixing the number of pixels in the three planes allow the generation of population based maps where the approximate distribution of distances, uncertainties can be assessed. The final projection images are shown with equal width and an aspect ratio corresponding to the mean structure based on all scans of all patients.

\section{Statistical analysis}

Differences between patient median DSC, MSD and HD values between CT-MR, MR-MR registrations and

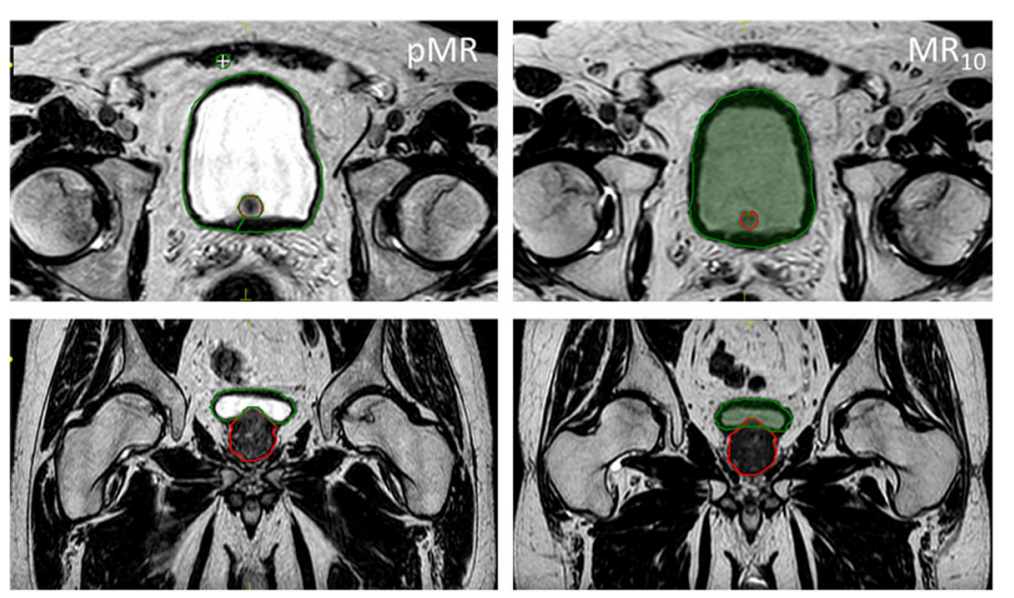

Fig. 3 Prostate (red) and bladder (green) delineated manually on pMR, shown in transverse and coronal view on the left. Note that the bladder and prostate do not occupy the same space. On the right these structures have been propagated onto $\mathrm{MR}_{10}$, and the bladder structure overlaps the prostate 
ground truth, as well as difference in structure volume between $\mathrm{pCT}$ and $\mathrm{pMR}$, were tested for statistical significance by Wilcoxon signed-rank tests at the $5 \%$ significance level.

\section{Results}

In total 672 structures were created successfully by DIR propagation. However, for $25 \%$ of the patients (3/12) the bladder was not propagated correctly because of the TPS' inability to deform pin hole structures (the TPS' best approximation of a donut structure) correctly, as illustrated in Fig. 3. All MR-MR propagated structures yielded higher population median DSC than CT-MR propagations when compared to ground truth delineations (Table $1 \mathrm{~A}$ ), implying that MR-MR DIR is more accurate. This was statistically significant for the prostate, SV, rectum, femoral heads and penile bulb.

Population median MSD values demonstrated better agreement with the ground truth for all deformed structures based on MR relative to CT (MR-MR values were $0.17-1.16 \mathrm{~mm}$ lower). Differences were statistically significant for all structures except CTV56 (Table 1 B).

Table 1 Population median DSC (A), MSD (B) and HD (C) for all patients of the 4 CT-MR propagations, 3 MR-MR propagations, intraobserver variations $(\mathrm{IOV})$ and their differences $(\Delta)$ are shown for each structure

\begin{tabular}{|c|c|c|c|c|c|c|c|c|c|}
\hline \multirow[t]{3}{*}{$A$} & \multicolumn{9}{|c|}{ Population median DSC } \\
\hline & \multirow[b]{2}{*}{ CT-MR } & \multirow[b]{2}{*}{ MR-MR } & \multirow[b]{2}{*}{ IOV } & \multicolumn{2}{|c|}{ CT-MR vs MR-MR } & \multicolumn{2}{|c|}{ CT-MR vs IOV } & \multicolumn{2}{|c|}{ MR-MR vs IOV } \\
\hline & & & & $\Delta$ & $p$-value & $\Delta$ & $p$-value & $\Delta$ & $p$-value \\
\hline Prostate & 0.84 & 0.90 & 0.92 & -0.07 & $<0.001$ & -0.08 & $<0.001$ & -0.02 & 0.01 \\
\hline SV & 0.68 & 0.76 & 0.81 & -0.08 & 0.001 & -0.3 & $<0.001$ & -0.05 & 0.05 \\
\hline CTV56 & 0.84 & 0.84 & 0.87 & 0.0 & 0.73 & -0.03 & 0.02 & -0.03 & 0.23 \\
\hline Rectum & 0.77 & 0.87 & 0.95 & -0.10 & $<0.001$ & -0.18 & $<0.001$ & -0.08 & 0.003 \\
\hline Bladder & 0.87 & 0.92 & 0.97 & -0.05 & 0.15 & -0.10 & $<0.001$ & -0.05 & $<0.001$ \\
\hline R fem. Head & 0.93 & 0.95 & 0.95 & -0.02 & $<0.001$ & -0.02 & 0.005 & -0.00 & 0.18 \\
\hline L fem. Head & 0.91 & 0.94 & 0.94 & -0.03 & 0.007 & -0.03 & 0.002 & 0.00 & 0.20 \\
\hline Penile Bulb & 0.64 & 0.79 & 0.85 & -0.15 & $<0.001$ & -0.21 & $<0.001$ & -0.06 & 0.08 \\
\hline \multirow[t]{3}{*}{ B } & \multicolumn{9}{|c|}{ Population median MSD [mm] } \\
\hline & & & & \multicolumn{2}{|c|}{ CT-MR vs MR-MR } & \multicolumn{2}{|c|}{ CT-MR vs IOV } & \multicolumn{2}{|c|}{ MR-MR vs IOV } \\
\hline & CT-MR & MR-MR & IOV & $\Delta$ & $p$-value & $\Delta$ & $p$-value & $\Delta$ & $p$-value \\
\hline Prostate & 1.60 & 1.00 & 0.88 & 0.60 & $<0.001$ & 0.72 & $<0.001$ & 0.12 & 0.15 \\
\hline SV & 1.48 & 1.17 & 0.86 & 0.31 & 0.002 & 0.62 & 0.005 & 0.31 & 0.13 \\
\hline CTV56 & 1.99 & 1.82 & 1.58 & 0.17 & 0.30 & 0.41 & $<0.001$ & 0.24 & 0.13 \\
\hline Rectum & 2.41 & 1.25 & 0.65 & 1.16 & 0.007 & 1.76 & $<0.001$ & 0.6 & 0.001 \\
\hline Bladder & 1.96 & 1.11 & 0.55 & 0.85 & 0.03 & 1.41 & $<0.001$ & 0.56 & $<0.001$ \\
\hline R fem. Head & 1.09 & 0.81 & 0.75 & 0.28 & 0.002 & 0.34 & 0.005 & 0.06 & 0.34 \\
\hline L fem. Head & 1.37 & 0.81 & 1.05 & 0.56 & 0.03 & 0.32 & 0.03 & -0.24 & 0.42 \\
\hline Penile Bulb & 1.61 & 0.89 & 0.74 & 0.72 & $<0.001$ & 0.87 & 0.002 & 0.15 & 0.13 \\
\hline \multirow[t]{3}{*}{ C } & \multicolumn{9}{|c|}{ Population median HD [mm] } \\
\hline & & & & \multicolumn{2}{|c|}{ CT-MR vs MR-MR } & \multicolumn{2}{|c|}{ CT-MR vs IOV } & \multicolumn{2}{|c|}{ MR-MR vs IOV } \\
\hline & CT-MR & MR-MR & IOV & $\Delta$ & $p$-value & $\Delta$ & $p$-value & $\Delta$ & $p$-value \\
\hline Prostate & 7.16 & 5.10 & 4.89 & 2.16 & 0.005 & 2.06 & $<0.001$ & 2.27 & 0.42 \\
\hline SV & 6.55 & 5.54 & 5.31 & 1.01 & 0.18 & 1.01 & 0.04 & 1.24 & 0.42 \\
\hline CTV56 & 14.92 & 12.77 & 11.55 & 2.15 & 0.34 & 2.15 & 0.03 & 3.37 & 0.42 \\
\hline Rectum & 12.36 & 8.89 & 7.65 & 3.47 & 0.38 & 3.47 & 0.06 & 4.71 & 0.47 \\
\hline Bladder & 10.88 & 5.71 & 4.05 & 5.17 & 0.38 & 5.17 & $<0.001$ & 6.83 & 0.01 \\
\hline R fem. Head & 4.96 & 4.77 & 4.41 & 0.13 & 0.38 & 0.19 & 0.42 & 0.55 & 0.62 \\
\hline L fem. Head & 4.98 & 4.75 & 5.21 & 0.23 & 0.30 & 0.23 & 0.85 & -0.23 & 0.85 \\
\hline Penile Bulb & 6.38 & 3.96 & 3.46 & 2.42 & $<0.001$ & 2.42 & 0.002 & 2.92 & 0.38 \\
\hline
\end{tabular}




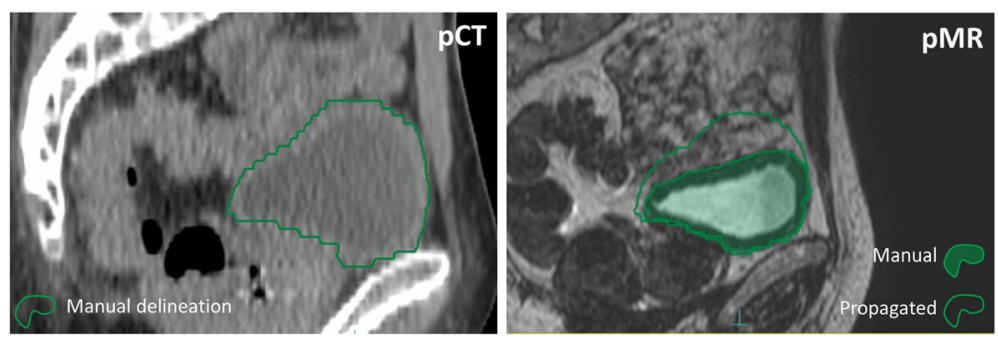

Fig. 4 The large bladder volume seen on PCT and deformed to pMR matched the bladder seen on pMR poorly. Generally, large differences were not well accounted for by the deformable structure propagation

Overall, CT-MR deformed structures showed inferior DSC and MSD compared to the intra-observer variation in contrast to MR-MR registration. MR-MR deformed structures generally showed population median DSC and MSD values in the same range as the intra-observer variations, but only statistically significant in prostate, bladder and rectum.

MR-MR DIR yielded smaller HD for all 8 investigated structures than CT-MR but were only statistically significant for prostate and penile bulb (Table $1 \mathrm{C}$ ).

Population median DSC and MSD showed statistically significant difference between CT-MR propagated contours and the intra-observer variation for all organs (Table 1 A). MR-MR was statistically similar to the intra observer variation in most cases (4 of 8 for DSC and 6 of 8 for MSD).

Visual inspection of the deformed structures and their source and destination image sets revealed that small differences between organs in the two image sets were generally well accounted for by the DIR algorithm. Contrarily, large differences were in most cases not at all compensated, as exemplified in Fig. 4. This effect impacted on the MSD and DSC values; deformable organs, such as the bladder and rectum, showed larger disagreements than rigid structures, e. g. the prostate and femoral heads. The difference in size between $\mathrm{pCT}$ or pMR and $\mathrm{MR}_{\mathrm{x}}$ (Table 2) potentially affects the resulting deformation quality. Large variations in volume were observed for bladder and rectum delineations. The prostate structure was systematically smaller when delineated on MR than on CT $(p<0.001)$.

Table 2 Median volumes, and their range in brackets, of prostate, bladder and rectum structures manually delineated on planning images. Statistical test of the difference between volumes on CT versus MR was performed and the result given as the $p$-value

\begin{tabular}{llll}
\hline Structure & $\vee(\mathrm{pCT})\left[\mathrm{cm}^{3}\right]$ & $\vee(\mathrm{pMR})\left[\mathrm{cm}^{3}\right]$ & $p$-value \\
\hline Prostate & $45.5(27.2-132,5)$ & $38.1(23.7-112.6)$ & $<0.001$ \\
Bladder & $186.5(85.8-460.5)$ & $174.3(57.7-534.4)$ & 0.25 \\
Rectum & $96.1(54.3-133.0)$ & $94.0(38.3-136.8)$ & 0.16 \\
\hline
\end{tabular}

In Fig. 5, the DSC and MSD were plotted against the ratio of structure volume on the planning image versus the ground truth on the daily image $\left(\mathrm{MR}_{\mathrm{x}}\right)$. For the prostate, only small deviations were observed between the volumes of the structure between the planning image and subsequent images, which translates to consistently high DSC and low MSD for all deformations. Still, a tendency of DSC being closer to 1 and low MSD values for ratios near 1 was observed.

A correlation between volume ratio and accuracy of propagated structures was seen for the bladder by the parabolic tendency in the plots with extrema approximately at volume ratio 1 (Fig. 6). A similar pattern was seen for rectum propagations, although less pronounced than for the bladder (Additional file 1: Figure A1).

The population difference between ground truth and deformed or re-delineated structures are visualized for the prostate in Fig. 7 . The projection images show that the delineation differences overall were larger for CTMR compared to MR-MR. Thus, the observed difference between the image modalities was not only related to specific volumes of the prostate, but also variation in delineations due to different image modalities. The largest error in DIR propagated prostate structures were seen in the anterior-cranial part of the gland and towards the rectum for CT-MR deformations (Fig. 7).

A similar pattern was observed in the population projection images for rectum (Fig. 8); overall, variations were larger for CT-based DIR with the largest differences towards the cranial boundary and the anterior wall. MR-MR DIR propagated structures were generally in good agreement, although cranially differences exceeded $3 \mathrm{~mm}$. The trend was similar for the intraobserver variation. Projection images of the remaining investigated structures showed similar patterns; the largest deviations between both CT-MR and MR-MR propagated structures and ground truth was seen at the cranial and caudal limits of CTV56 (Additional file 1: Figure B1). The seminal vesicles showed the largest deviations anteriorly for CT-MR, but cranially for MR-MR (Additional file 1: Figure B2). The penile bulb propagations showed the largest deviations anteriorly for CT- 

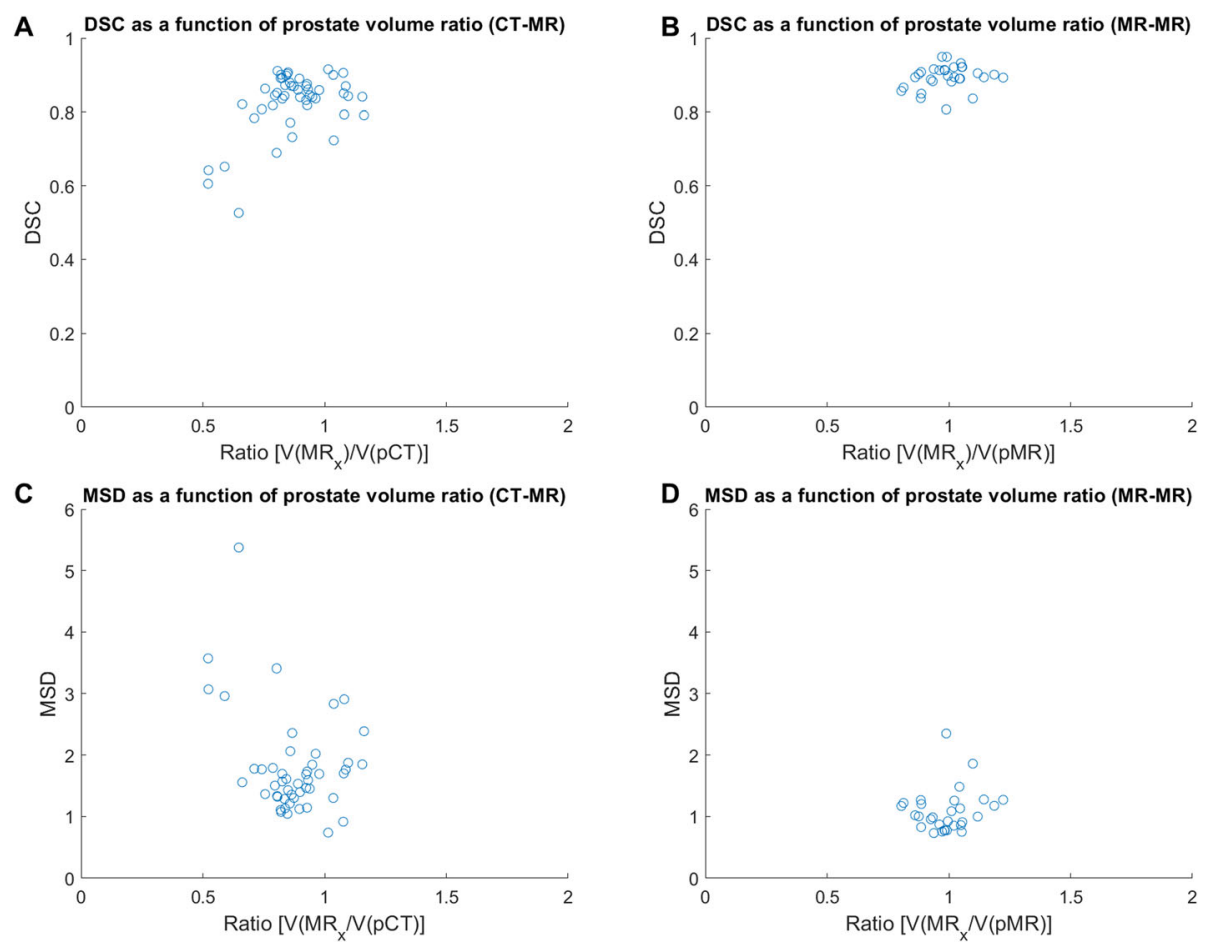

Fig. 5 DSC and MSD plotted against the ratio of ground truth prostate volumes of planning images (pCT or pMR) and the image of the day $\left(M R_{x}\right)$

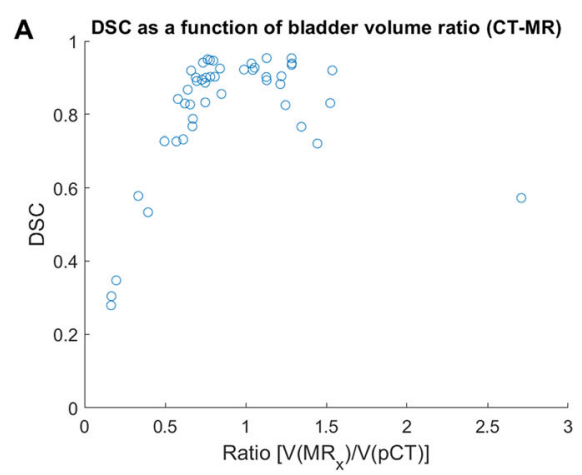

C

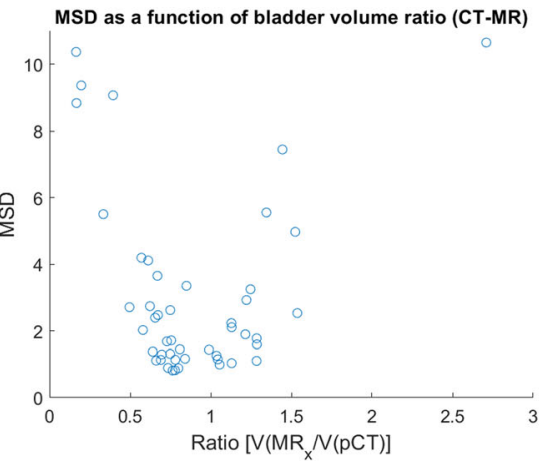

B DSC as a function of bladder volume ratio (MR-MR)

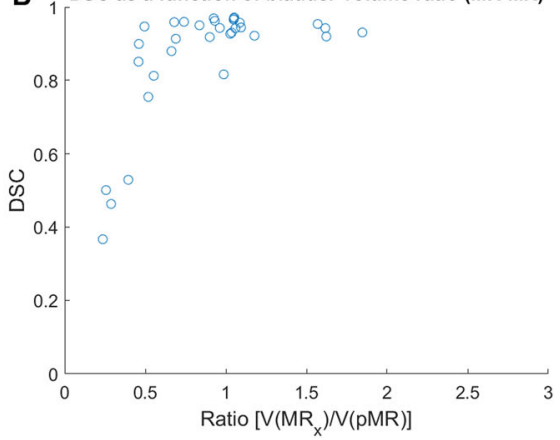

D MSD as a function of bladder volume ratio (MR-MR)

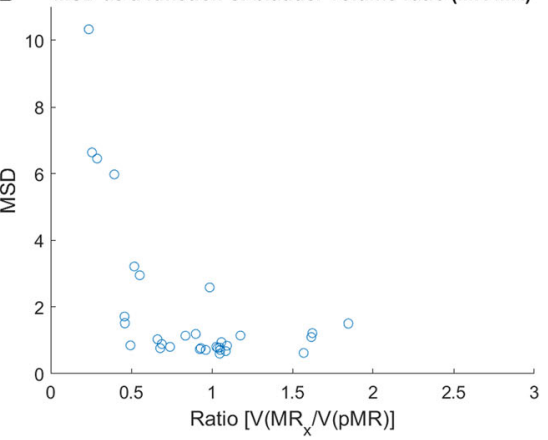

Fig. 6 DSC and MSD plotted against the ratio of ground truth bladder volumes of planning images (pCT or pMR) and the image of the day (MR $)$ 


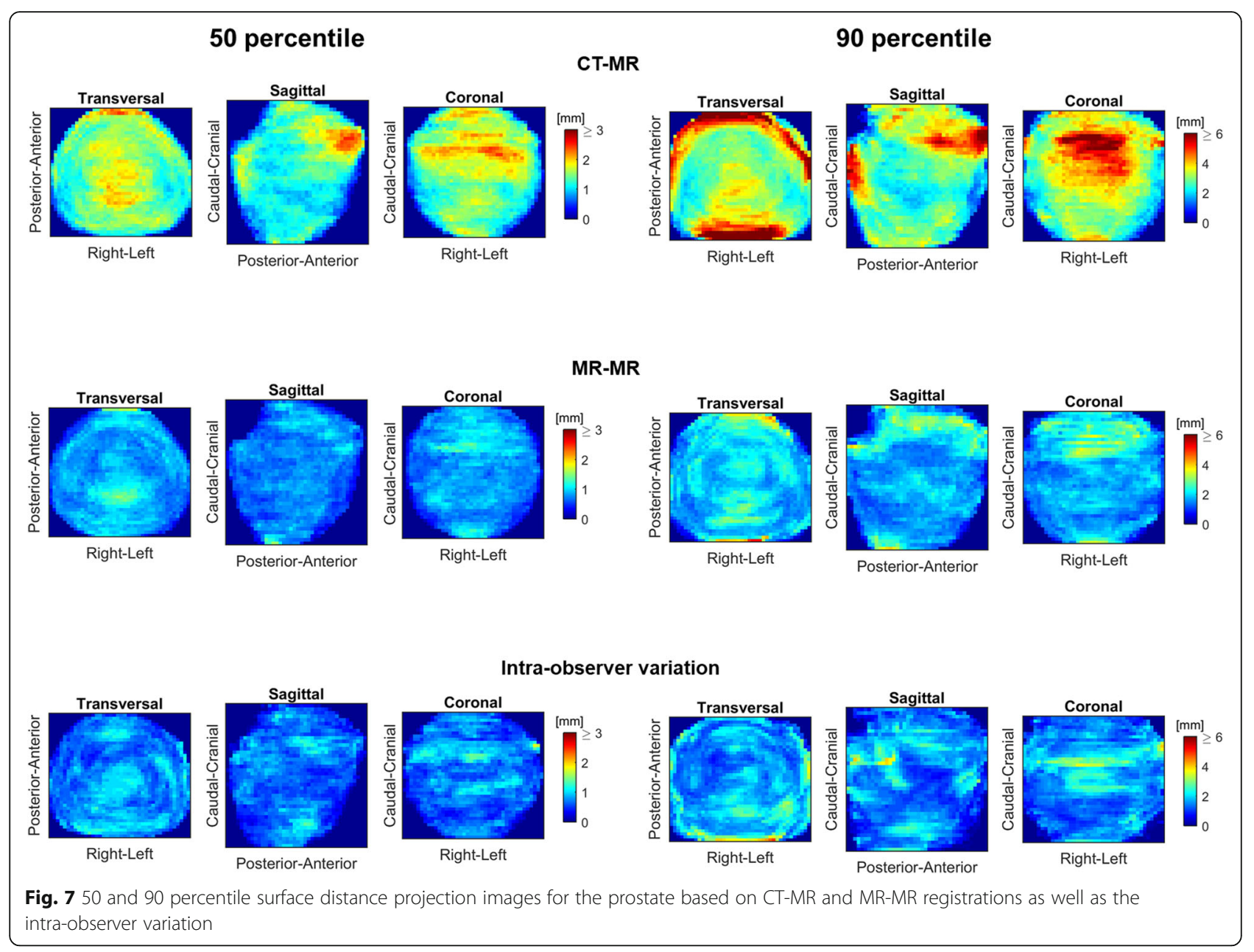

MR, while MR-MR propagations were similar to the intra-observer variation (Additional file 1: Figure B6).

\section{Discussion}

This validation study has shown that intra-modal MRMR image deformation is almost comparable in accuracy to the intra-observer variation of manual delineations. Inter-modal CT-MR DIR was less accurate and thus not ideal for clinical use. CT-MR deformations were less accurate than the intra-observer variation and therefore will require more time for manual editing. This is problematic for online treatment adaptation due to the inconsistency in target and OAR definition but also due to the risk of internal organ motion increases. Because MR-MR deformations were similar in accuracy to intraobserver variations and thus require less manual corrections, the delineating clinician might have a better starting point when defining the target and OAR, and the risk of errors in delineation decreases.

This result is similar to what has been shown to be the case for manual delineations: MR provides higher consistency of the delineations. In this light, the workflow using the $\mathrm{pCT}$ as the source for DIR to the session MR, as proposed as standard by the MRL vendor, might be suboptimal. Instead, a workflow using the pMR as the source would provide a set of propagated contours that better represent their actual shape and position. Hence, time required to review and revise while the patient is on the couch, is minimized. From the population surface distance projection images, it appears that some DIR propagation errors are systematic: Both for CT-MR and MR-MR the prostate differs mainly in the most anterior and cranial region, CTV56 at the caudal and cranial boundaries. Variations in bladder and rectum filling require corrections cranially and the penile bulb generally requires corrections anteriorly. Comparison of the 90 percentile surface distance projection images for MR-MR to the related intra-observer variation reveals that regions of large DIR uncertainty are also regions of large intra-observer variation, thus probably regions for which it inherently is difficult to define the "ground truth" of the delineation. Similar observations were made for rectum (Fig. 5), CTV56 (Additional file 1: Figure B1) and penile bulb (Additional 


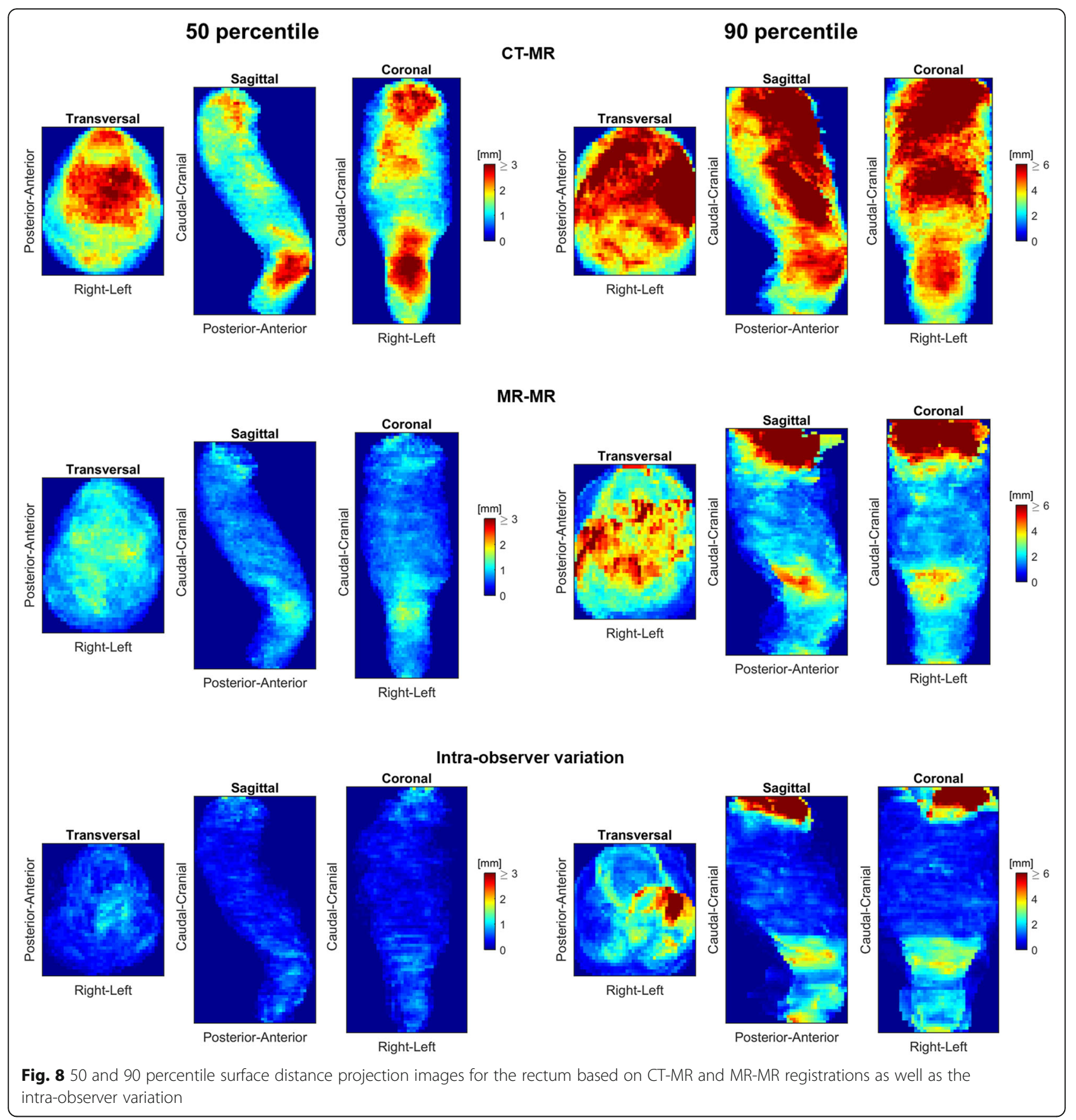

file 1: Figure B6). Since some structures appear more difficult to deform accurately for the DIR algorithm, it might produce better results if future algorithms include organ specific information, thereby allowing, e. g., larger variations in the cranio-caudal direction for the bladder. However, with the currently available DIR algorithm, the correlation found between large differences in the bladder volume on the planning image and daily image, indicates that minimizing the variation between the source image and images of the day may be appropriate, e. g. by using a drinking protocol. The current results indicates that only for volume ratios in the range of $0.8-1.2$ result in reasonably accurate DIR structure propagations. Neither the CT-MR nor the MR-MR DIR was able to account for large volume changes.

It should be noted that in this study, the slice thickness of CT $(3 \mathrm{~mm})$ and MR $(1 \mathrm{~mm})$ differ. This means that by default there can be a difference between delineations cranially and caudally of up to $2 \mathrm{~mm}$ due to this fact alone. For structures of little extension in the 
caudo-cranial direction, but large in other directions this could have a non-negligible impact, favoring MR-MR DIR in this study. Equally, the difference in the pixel size between CT and MR could mean differences of up to $0.5 \mathrm{~mm}$ right-left and anterior-posterior directions. However, the population surface distance projection images of this study do not indicate that the difference in slice thickness severely impacts these results, as the geometric distribution of differences is the same for CT-MR as MR-MR. E.g. the difference between ground truth and DIR propagated contours is not increased in the most cranial and caudal regions of rigid structures such as the femoral heads for the CT-MR registrations compared to the more central regions (see Figure B4 and B5 in the Additional file 1).

Image quality itself could affect image registration and resulting structure propagations. In the current study, MR image acquisition during the course of treatment was made on a diagnostic MR scanner, rather than on the MR Linac itself. Although the scan sequence used were set up as closely as possible to the proposed clinical settings on the MRL, the acquired image quality is not identical to that of the MRL, as much of the hardware is designed differently, e. g. the magnet, gradient magnets and coils. A detailed comparison of image quality between the two scanners is beyond the scope of the current study. However, it has previously been demonstrated that the geometric fidelity of the scanners are similar [28].

For a workflow using MR as the primary image set for dose planning, electron densities required for dose calculations must be generated either based on a pCT or alternatively from a MR based pseudo CT in order to apply a MR-only planning workflow [29-31]. ART can also be performed using $\mathrm{CBCT}$, which does provide ED information, which, with correct adjustments, can provide the basis for precise dose calculations [32-34]. With current standard CBCT technology, the accuracy of CT DIR to CBCT is similar to that of the inter-observer variation [35, 36]. Future publications will document if new $\mathrm{CBCT}$ based with iterative image reconstruction technologies are able to increase deformation accuracy.

The organs at risk evaluated in this study are relevant for other pelvic indications than prostate cancer. Further studies will have to assess whether the DIR accuracy observed here also apply in other anatomical regions. Systematic shrinkage or swelling of organs is not expected for prostate cancer patients over the course of RT [37]. Therefore, continuously using the $\mathrm{pCT}$ and $\mathrm{pMR}$, rather than successively using the latest acquired MR, should not affect the resulting overall accuracy of the DIR. In other indications, e.g. head and neck cancers and lung cancers, tumor shrinkage has been observed [38, 39]. For such indications, it might be appropriate to successively use the last acquired MR for DIR for daily plan adaptation.

\section{Conclusion}

This study has shown that for high risk prostate cancer patients to be treated with an adapted plan on the Unity MRL, structures propagated from planning scan images to online daily MR images need manual editing, whether they were propagated from CT or MR. But the current results show that MR-MR propagated structures require fewer corrections and are therefore preferred for clinical use, as the online planning time may decrease and delineation accuracy increase compared to a CT-MR workflow. Thus, a clinical MR-MR workflow has been implemented locally.

\section{Supplementary information}

Supplementary information accompanies this paper at https://doi.org/10. 1186/s13014-020-1482-y.

Additional file 1. High resolution versions of images in the appendices are provided in the following files.

\section{Abbreviations}

ART: Adaptive radiotherapy; CBCT: Cone beam computed tomography; $\mathrm{CT}$ : Computed tomography; CTV: Clinical target volume; DIR: Deformable image registration; DSC: Dice similarity coefficient; FOV: Field of view; HD: Hausdorff distance; IOV: Intra-observer variation; L: Left; MR: Magnetic resonance; MRI : Magnetic resonance imaging; MRL: Magnetic resonance linac; MSD: Mean surface distance; OAR: Organ at risk; pCT: Planning computed tomography; pMR: Planning magnetic resonance; R: Right; RT: Radiotherapy; RTT: Radiation therapist; SV: Seminal vesicles; TE: Time to echo; TPS: Treatment planning system; TR: Time to repeat; VMAT: Volumetric modulated arc therapy

\section{Acknowledgements}

Not applicable.

\section{Authors' contributions}

The study was designed by UB, CB, LD and RLC. Data was generated by LD and RLC. UB, CB and RLC analysed the data. All authors contributed to the writing of the manuscript and approved the final version.

\section{Funding}

RLC was supported by scholarships from the University of Southern Denmark and Odense University Hospital. All authors acknowledge support from MANTRA (New MAgNetic resonance Technology for Response Adapted radiotherapy), a Frontline research center based at Odense University Hospital, Denmark and AgeCare (Academy of Geriatric Cancer Research), an international research collaboration based at Odense University Hospital, Denmark.

None of the funding bodies have been involved in the design of the study, data collection, analysis or interpretation of data.

Availability of data and materials

Please contact author for data requests.

\section{Ethics approval and consent to participate}

This trail is registered and approved by The Committee for Scientific Ethics of the Region of Southern Denmark under ID S-20170143. All included patients have given signed consent prior to their participation.

\section{Consent for publication}

Written consent to enter the study and publication of anonymized data were obtained from all participants. 


\section{Competing interests}

The authors declare that they have no competing interests.

\section{Author details}

'Department of Clinical Research, University of Southern Denmark, Winsløwparken 19 3. Sal, 5000 Odense C, Denmark. ${ }^{2}$ Laboratory of Radiation Physics, Department of Oncology, Odense University Hospital, Kløvervænget 19, Indgang 85, Pavillion, Stuen, 5000 Odense C, Denmark. ${ }^{3}$ Department of Oncology, Odense University Hospital, Kløvervænget 19 Indgang 85 Pavillion, 1. sal, 5000 Odense C, Denmark.

Received: 2 August 2019 Accepted: 30 January 2020 Published online: 07 February 2020

\section{References}

1. Muren LP, Thwaites DI. The on-going quest for treatment precision and conformality in radiotherapy. Radiother Oncol. 2013;109:337-41.

2. Pathmanathan AU, van As NJ, Kerkmeijer LGW, Christodouleas J, Lawton CAF, Vesprini D, et al. Magnetic resonance imaging-guided adaptive radiation therapy: a "game changer" for prostate treatment? Int J Radiat Oncol Biol Phys. 2018;100:361-73.

3. Thor M, Petersen JBB, Bentzen L, Hoyer M, Muren LP. Deformable image registration for contour propagation from $C T$ to cone-beam $C T$ scans in radiotherapy of prostate cancer. Acta Oncol. 2011;50:918-25.

4. Ahmad R, Bondar L, Voet P, Mens JW, Quint S, Dhawtal G, et al. A margin-of-the-day online adaptive intensity-modulated radiotherapy strategy for cervical cancer provides superior treatment accuracy compared to clinically recommended margins: a dosimetric evaluation. Acta Oncol. 2013;52:1430-6.

5. Kontaxis C, Bol GH, Lagendijk JJ, Raaymakers BW. Towards adaptive IMRT sequencing for the MR-linac. Phys Med Biol. 2015;60:2493-509.

6. Mutic S, Dempsey JF. The ViewRay system: magnetic resonance-guided and controlled radiotherapy. Semin Radiat Oncol. 2014;24:196-9.

7. Lagendijk JJ, Raaymakers BW, van Vulpen M. The magnetic resonance imaging-linac system. Semin Radiat Oncol. 2014;24:207-9.

8. Sander L, Langkilde NC, Holmberg M, Carl J. MRI target delineation may reduce long-term toxicity after prostate radiotherapy. Acta Oncol. 2014; 53:809-14.

9. Kontaxis C, Bol GH, Kerkmeijer LGW, Lagendijk JJW, Raaymakers BW. Fast online replanning for interfraction rotation correction in prostate radiotherapy. Med Phys. 2017:44:5034-42.

10. Raaymakers BW, Jurgenliemk-Schulz IM, Bol GH, Glitzner M, Kotte A, van Asselen B, et al. First patients treated with a 1.5 T MRI-Linac: clinical proof of concept of a high-precision, high-field MRI guided radiotherapy treatment. Phys Med Biol. 2017;62:L41-50.

11. Bertelsen AS, Schytte T, Moller PK, Mahmood F, Riis HL, Gottlieb KL, et al. First clinical experiences with a high field 1.5 T MR linac. Acta Oncol. 2019:1-6.

12. Acharya S, Fischer-Valuck BW, Kashani R. Online magnetic resonance image guided adaptive radiation therapy: First clinical applications (vol 94, pg 394, 2016). Int J Radiat Oncol. 2016;96:243.

13. Poon M, Holborn C, Cheng KF, Fung WWK, Chiu G. Evaluation of deformed image-based dose calculations for adaptive radiotherapy of nasopharyngeal carcinoma. Med Dosim. 2017;42:273-81.

14. Oh S, Kim S. Deformable image registration in radiation therapy. Radiat Oncol J. 2017:35:101-11.

15. Thornqvist S, Petersen JB, Hoyer M, Bentzen LN, Muren LP. Propagation of target and organ at risk contours in radiotherapy of prostate cancer using deformable image registration. Acta Oncol. 2010;49:1023-32.

16. Wang H, Garden AS, Zhang L, Wei X, Ahamad A, Kuban DA, et al. Performance evaluation of automatic anatomy segmentation algorithm on repeat or fourdimensional computed tomography images using deformable image registration method. Int J Radiat Oncol Biol Phys. 2008;72:210-9.

17. Beasley WJ, McWilliam A, Slevin NJ, Mackay Rl, van Herk M. An automated workflow for patient-specific quality control of contour propagation. Phys Med Biol. 2016;61:8577-86.

18. Kieselmann JP, Kamerling CP, Burgos N, Menten MJ, Fuller CD, Nill S, et al. Geometric and dosimetric evaluations of atlas-based segmentation methods of MR images in the head and neck region. Phys Med Biol. 2018;63:145007.

19. Brock KK. Deformable registration accuracy C. results of a multi-institution deformable registration accuracy study (MIDRAS). Int J Radiat Oncol Biol Phys. 2010;76:583-96.
20. Smith WL, Lewis C, Bauman G, Rodrigues G, D'Souza D, Ash R, et al. Prostate volume contouring: a 3D analysis of segmentation using 3DTRUS, CT, and MR. Int J Radiat Oncol Biol Phys. 2007;67:1238-47.

21. Khoo EL, Schick K, Plank AW, Poulsen M, Wong WW, Middleton M, et al. Prostate contouring variation: can it be fixed? Int J Radiat Oncol Biol Phys. 2012;82:1923-9.

22. Rasch C, Barillot I, Remeijer P, Touw A, van Herk M, Lebesque JV. Definition of the prostate in CT and MRI: a multi-observer study. Int J Radiat Oncol Biol Phys. 1999;43:57-66.

23. McErlean A, Panicek DM, Zabor EC, Moskowitz CS, Bitar R, Motzer RJ, et al. Intra- and interobserver variability in CT measurements in oncology. Radiology. 2013;269:451-9.

24. Lawton CA, Michalski J, El-Naqa I, Buyyounouski MK, Lee WR, Menard C, et al. RTOG GU radiation oncology specialists reach consensus on pelvic lymph node volumes for high-risk prostate cancer. Int J Radiat Oncol Biol Phys. 2009;74:383-7.

25. Dice LR. Measures of the amount of ecologic association between species. Ecology. 1945;26:297-302.

26. Zukauskaite R, Brink C, Hansen CR, Bertelsen A, Johansen J, Grau C, et al. Open source deformable image registration system for treatment planning and recurrence $C T$ scans : validation in the head and neck region. Strahlenther Onkol. 2016;192:545-51.

27. Kumarasiri A, Siddiqui F, Liu C, Yechieli R, Shah M, Pradhan D, et al. Deformable image registration based automatic CT-to-CT contour propagation for head and neck adaptive radiotherapy in the routine clinical setting. Med Phys. 2014;41:121712.

28. Jensen HR, Thomsen JB, Christiansen RL, Bertelsen A, Bernchou U, Brink C, et al. Comparison of Geometrical Distortion in MR Images from of Low-Field and High-Field MR-Linac. Int J Radiat Oncol. 2019;105:S239-S.

29. Christiansen $\mathrm{RL}$, Jensen $H R$, Brink C. Magnetic resonance only workflow and validation of dose calculations for radiotherapy of prostate cancer. Acta Oncol. 2017:56:787-91.

30. Tyagi N, Fontenla S, Zelefsky M, Chong-Ton M, Ostergren K, Shah N, et al. Clinical workflow for MR-only simulation and planning in prostate. Radiat Oncol. 2017;12:119.

31. Tenhunen M, Korhonen J, Kapanen M, Seppala T, Koivula L, Collan J, et al. MRI-only based radiation therapy of prostate cancer: workflow and early clinical experience. Acta Oncol. 2018;57:902-7.

32. Fotina I, Hopfgartner J, Stock M, Steininger T, Lutgendorf-Caucig C, Georg D. Feasibility of CBCT-based dose calculation: comparative analysis of $\mathrm{HU}$ adjustment techniques. Radiother Oncol. 2012;104:249-56.

33. Thing RS, Bernchou U, Mainegra-Hing E, Hansen O, Brink C. Hounsfield unit recovery in clinical cone beam $C T$ images of the thorax acquired for image guided radiation therapy. Phys Med Biol. 2016;61:5781-802.

34. Thing RS, Bernchou U, Hansen O, Brink C. Accuracy of dose calculation based on artefact corrected cone beam CT images of lung cancer patients. Phys Im Radiat Oncol. 2017;1:6-11.

35. Rigaud B, Simon A, Castelli J, Lafond C, Acosta O, Haigron P, et al. Deformable image registration for radiation therapy: principle, methods, applications and evaluation. Acta Oncol. 2019;58:1225-37.

36. Riegel AC, Antone JG, Zhang H, Jain P, Raince J, Rea A, et al. Deformable image registration and interobserver variation in contour propagation for radiation therapy planning. J Appl Clin Med Phys. 2016;17:347-57.

37. Scaife J, Harrison K, Romanchikova M, Parker A, Sutcliffe M, Bond S, et al. Random variation in rectal position during radiotherapy for prostate cancer is two to three times greater than that predicted from prostate motion. $\mathrm{Br}$ J Radiol. 2014;87:20140343.

38. Brouwer CL, Steenbakkers RJHM, Langendijk JA, Sijtsema NM. Identifying patients who may benefit from adaptive radiotherapy: does the literature on anatomic and dosimetric changes in head and neck organs at risk during radiotherapy provide information to help? Radiother Oncol. 2015;115:285-94.

39. Kilburn JM, Lucas JT, Soike MH, Ayala-Peacock D, Kuremsy JG, Blackstock A, et al. Which Lung Tumors Shrink During Fractionated Radiation and How Does This Influence Outcome?: Appropriately Selecting Tumor and Treatment Characteristics for Adaptive Radiation Therapy Protocols. Int J Radiat Oncol. 2014;90:S53-S.

\section{Publisher's Note}

Springer Nature remains neutral with regard to jurisdictional claims in published maps and institutional affiliations. 